\title{
Translocation of the conus medullaris during dynamic lumbosacral magnetic resonance imaging in dogs
}

\author{
Jihye Nam DVM, MS \\ Kyuyong Kang DVM, PhD \\ Kyeonga Kim DVM, MS \\ Jeongwoo Choi DVM, MS \\ Mincheol Choi DVM, PhD \\ Junghee Yoon DVM, PhD
}

Received January 22, 2020

Accepted October 8, 2020

From the Department of Clinical Sciences, College of Veterinary Medicine, and Research Institute for Veterinary Science, Seoul National University, Seoul 08826, Republic of Korea.

Address correspondence to Dr. Yoon (heeyoon@snu. ac.kr).

\begin{abstract}
OBJECTIVE
To investigate the change in the lumbosacral angle $(\triangle \mathrm{LSA})$ and conus medullaris (CM) displacement in healthy dogs undergoing dynamic MRI with changes in the posture of their pelvic limbs from neutral posture to flexion or extension posture and to evaluate for potential correlation between $\triangle \mathrm{LSA}$ and $\mathrm{CM}$ displacement.
\end{abstract}

\section{ANIMALS \\ 9 healthy adult Beagles.}

\section{PROCEDURES}

Dogs underwent dynamic MRI with their pelvic limbs positioned in neutral, flexion, and extension postures. From T2-weighted sagittal midline plane MRI images, 2 observers measured the lumbosacral angle and CM location in duplicate for each posture for each dog. Intra- and interobserver agreement was assessed, and the Spearman rank correlation coefficient $(\rho)$ was used to assess for potential correlation between $\triangle \mathrm{LSA}$ and CM displacement for changes in pelvic limb posture from neutral to flexion or extension.

\section{RESULTS}

Overall, the mean $\triangle \mathrm{LSA}$ and CM displacement for changes in posture were $23^{\circ}$ and $9.09 \mathrm{~mm}$ (caudal displacement) for the change from neutral to flexion posture, $8.4^{\circ}$ and $-2.5 \mathrm{~mm}$ (cranial displacement) for the change from neutral to extension posture, and $32.2^{\circ}$ and $11.64 \mathrm{~mm}$ (caudal displacement) for the change from extension to flexion posture. The $\Delta$ LSA strongly correlated ( $\rho=0.705 ; 95 \% \mathrm{Cl}, 0.434$ to 0.859 ) with displacement of the CM.

\section{CONCLUSIONS AND CLINICAL RELEVANCE}

The use of dynamic MRI, compared with conventional MRI, will better help to characterize clinically normal and abnormal features of the lumbosacral region of the vertebral column and associated spinal cord during postural changes. Further, when limited translocation of the CM is evident on dynamic MRI, veterinarians should suspect underlying lumbosacral pathophysiologic processes or anatomic abnormalities. (Am J Vet Res 2021;82:554-559)
$\mathrm{I}^{\mathrm{n}}$

T dogs, the lumbosacral region of the vertebral column is highly mobile, and degenerative disease will likely occur in this region when instability exists. ${ }^{1,2}$ In this same region, the spinal cord tapers to an end, referred to as the CM, and beyond which the filum terminale and cauda equina continue caudally through the vertebral column. ${ }^{1,3,4}$ Lumbosacral disease and cauda equina syndrome are general terms for any disease or abnormality that occurs in the

\begin{tabular}{|c|c|}
\hline ABBRE & IONS \\
\hline CM & Conus medullaris \\
\hline CM:L7 & $\begin{array}{l}\text { Conus medullaris displacement-to-L7 vertebral } \\
\text { length ratio }\end{array}$ \\
\hline $\mathrm{CM}: \Delta \mathrm{LSA}$ & $\begin{array}{l}\text { Conus medullaris displacement-to-change in } \\
\text { lumbosacral angle ratio }\end{array}$ \\
\hline$\Delta \mathrm{LSA}$ & Change in lumbosacral angle \\
\hline ICC & Intraclass correlation coefficient \\
\hline IVD & Intervertebral disk \\
\hline LSA & Lumbosacral angle \\
\hline SLR & Straight leg raise \\
\hline T2WI & T2-weighted image \\
\hline
\end{tabular}

lumbosacral region of the vertebral column, including congenital deformities, lumbosacral stenosis, IVD extrusion or luxation, diskospondylitis, inflammation, neoplasia, and vertebral fractures, and the underlying condition may be difficult to diagnose when neurologic and orthopedic disorders occur concomitantly. ${ }^{1,3-5}$

In people, the $\mathrm{CM}$ has a relatively consistent location, size, and shape ${ }^{6}$; however, in dogs, the size of the dog and vertebral column influence the locations of the CM and cauda equina.7.8 This variation in dogs further contributes to difficulties in diagnosing underlying conditions, such as tethered cord syndrome and congenital malformation of the filum terminale.-11 Although tethered cord syndrome can be diagnosed in people on the basis of the CM terminating distal to L2 (versus at the level of L1 for unaffected individuals), ${ }^{10,12}$ diagnosing tethered cord syndrome in dogs is challenging because the location of the CM varies in dogs and because of limitations with conventional static MRI. ${ }^{10}$ 
Also in people, studies ${ }^{13-18}$ show that CM displacement may be detected with the SLR test, during which each pelvic limb is individually positioned with the knee joint in extension to keep the leg straight while the hip joint is passively flexed by an examiner lifting the straight leg at the ankle joint. When this anterior postural change of the pelvic limb induces back pain, the SLR test result is considered positive and suggests the presence of restriction or displacement of the CM or another lumbosacral abnormality (eg, lumbosacral IVD disease). ${ }^{13-18}$

Evaluation of the lumbosacral region with MRI during dynamic posture changes is also a useful method for assessing neuromechanical impairment in people with sciatica. ${ }^{13-18}$ In veterinary medicine, a case report ${ }^{10}$ on dogs for which pelvic limb posture was changed during dynamic lumbosacral MRI shows no change in the position of the CM in a dog with tethered cord syndrome but a slight change in position of the CM in a dog with L1-2 IVD extrusion. However, to our knowledge, the effect of postural change on CM location within the vertebral column has not been explored in healthy dogs.

The aims of the study reported here were to investigate the $\triangle$ LSA and CM displacement in healthy dogs undergoing dynamic MRI with changes in the posture of their pelvic limbs from neutral posture to flexion or extension and to evaluate for potential correlations between $\triangle \mathrm{LSA}$ and CM displacement. We hypothesized that the CM displacement would correlate positively with the $\triangle \mathrm{LSA}$.

\section{Materials and Methods}

\section{Animals}

For this prospective study, 9 healthy Beagles were enrolled, and each dog underwent physical, neurologic, and radiographic examinations. For inclusion, dogs had to have been healthy with no signs of lameness, back pain, neurologic abnormality, or axial skeletal abnormality. The study was approved by the Institutional Animal Care and Use Committee of Seoul National University (approval No. SNU-190624-3).

\section{Anesthesia}

Food was withheld for 12 to 18 hours before the dogs underwent general anesthesia for dynamic MRI. For each dog, a 24-gauge catheter was aseptically placed in a cephalic vein, sedation was provided with acepromazine $(0.01 \mathrm{mg} / \mathrm{kg}, \mathrm{IV})$, general anesthesia was induced with alfaxalone $(2.0 \mathrm{mg} / \mathrm{kg}$, IV), an endotracheal tube was placed, and general anesthesia was maintained with isoflurane delivered in oxygen. During anesthesia and MRI, patient monitoring for each dog included ECG, pulse oximetry, end-tidal carbon dioxide concentration, minimum alveolar concentration of isoflurane, indirect blood pressure, and rectal temperature. Fluid therapy with sterile saline $(0.9 \% \mathrm{NaCl})$ solution $(2.5 \mathrm{~mL} / \mathrm{kg} / \mathrm{h}$, IV) was administered throughout the anesthetic event. No contrast medium was administered.

\section{Dynamic MRI}

A 0.3-T permanent MRI scanner ${ }^{a}$ with a head coil $^{\mathrm{b}}$ was used to obtain transverse and sagittal midline plane T2WIs of the lumbosacral regions of the vertebral column from L1 through Cd1 of each dog. The MRI sequence parameters for the sagittal plane T2WIs were repetition time of 1,729 milliseconds, echo time of 116 milliseconds, flip angle (rotation of the net magnetization vector by a radiofrequency pulse relative to the main magnetic field) of $120^{\circ}, 8$ acquisitions, slice thickness of $3.0 \mathrm{~mm}$, slice interval of $3.3 \mathrm{~mm}$, field of view of $220 \times 220 \mathrm{~mm}$, matrix (the numbers of row and columns in an image that make up the pixel) of $224 \times 128$, and scan duration of 14 minutes and 48 seconds. The MRI parameters for the transverse plane T2WIs were repetition time of 1,125 milliseconds, echo time of 140 milliseconds, flip angle of $90^{\circ}, 6$ acquisitions, slice thickness of $3.0 \mathrm{~mm}$, slice interval of $3.5 \mathrm{~mm}$, field of view of 220 $\times 220 \mathrm{~mm}$, matrix of $224 \times 128$, and scan duration of 16 minutes and 47 seconds.

While anesthetized, each dog underwent dynamic MRI positioned in dorsal recumbency and imaged in each of the 3 evaluated pelvic limb postures: neutral, flexion, and extension, in that order. The neutral posture was obtained by flexing the hip joints and stifle joints without any fixation (ie, frog-leg position). The flexion posture was obtained with flexion of the hip joints, extension of the stifle joints, and flexion of the tarsal joints, which were then held with elastic straps secured to the MRI table to maintain the pelvic limbs in this cranially directed posture. The extension posture was obtained with extension of the hip joints, stifle joints, and tarsal joints caudally, and the hind feet were restrained to the MRI table with elastic straps. After MRI, the administration of isoflurane was discontinued, and dogs were allowed to spontaneously breathe room air. Fluid therapy and patient monitoring with ECG and pulse oximetry continued through recovery until dogs were extubated.

\section{T2WI analysis}

All MRI images were analyzed with image analysis software. ${ }^{c}$ From the sagittal midline images obtained of each dog in each posture, the LSA and CM position were both measured twice by 2 observers to obtain data sets of 4 measurements per dog per posture. For each dog, a set of the same sagittal midline T2WIs of the dog with its pelvic limbs positioned in neutral, flexion, and extension postures, in that order, was provided to each of the observers in random order among the image sets for all 9 dogs.

\section{LSA}

The LSA was measured in the L7-S1 IVD space as the dorsal angle between a line drawn parallel to the craniocaudal horizontal axis of L7 and a similarly drawn line for S1 (Figure I). The $\Delta$ LSAs for each dog in each posture were determined by comparing the LSA measurements on T2WIs obtained with the dog's 

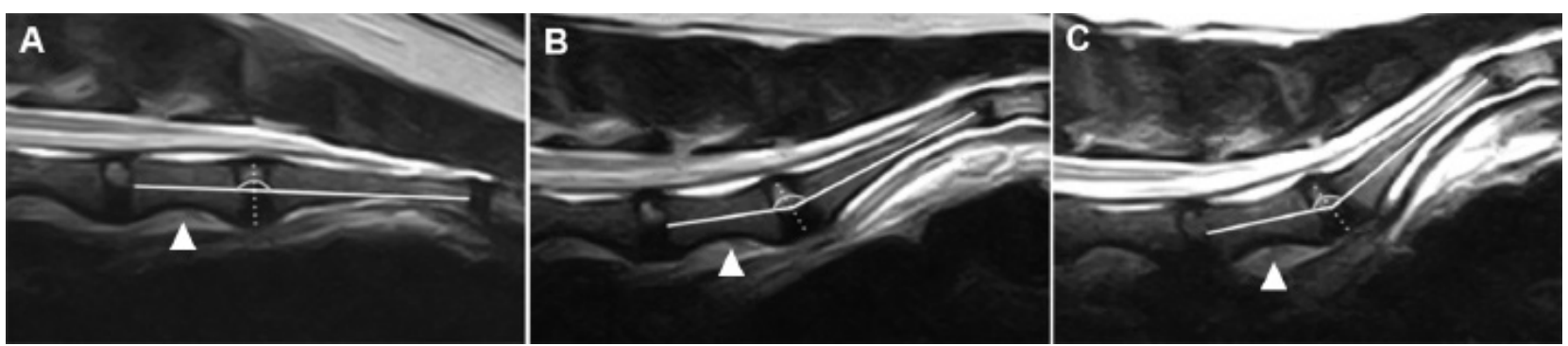

Figure I-Representative sagittal midline plane T2WIs of I of 9 healthy dogs that underwent dynamic MRI of the lumbosacral region of their vertebral column with their pelvic limbs positioned in flexion $(A)$, neutral $(B)$, and extension (C) postures, showing anatomic landmarks used in assessing the dog's LSA (arc), which was measured in the L7-SI IVD space where a line drawn parallel to the craniocaudal horizontal axis of L7 (arrowhead) and a similarly drawn line for SI transect (dotted line). For this dog, the LSA is $178.5^{\circ}$ for flexion posture (A), $157.0^{\circ}$ for neutral posture (B), and $149.4^{\circ}$ for extension posture (C). In all images, the dog's head is toward the left and dorsal is toward the top.
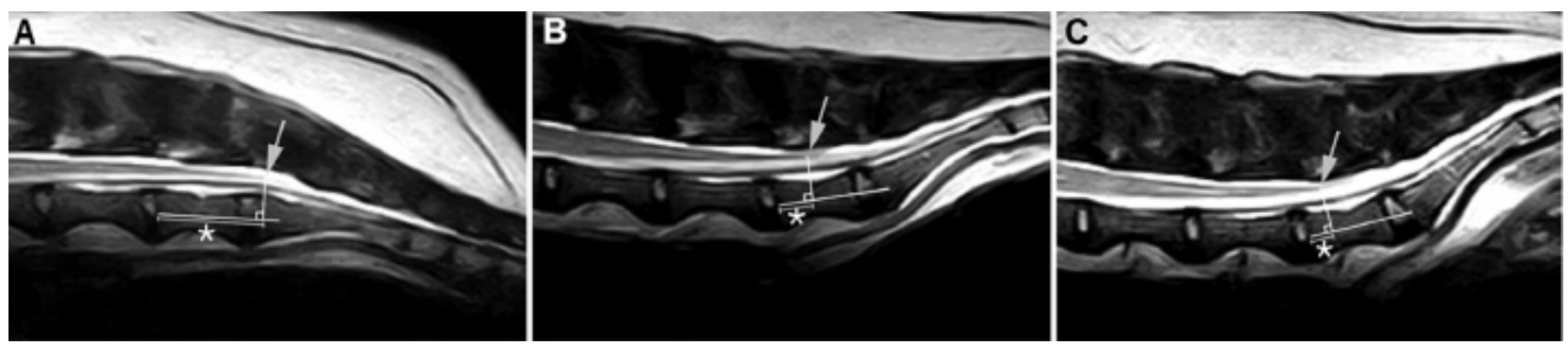

Figure 2-Representative sagittal midline plane T2Wls of I of the 9 dogs described in Figure I, showing the measurements (asterisks) of the location of the CM (arrows) relative to the level of the center of the cranial endplate of L7 that serves as the reference point and with the dog's pelvic limbs positioned in flexion (A), neutral (B), or extension (C) postures. In each image, a longer horizontal line passes through the centers of the cranial and caudal endplates of L7; a perpendicular line extends dorsally to the caudalmost aspect of the CM, determined as the point where the signal intensity of the spinal cord appeared hypointense, compared with the hyperintense signal of the CSF; and the shorter horizontal line represents the distance between the level of the cranial endplate of L7 and the level of the caudal tip of the CM, which is the CM location. In this dog, the CM location for each posture is $22.5 \mathrm{~mm}$ for flexion (A), $11.8 \mathrm{~mm}$ for neutral (B), and $9.6 \mathrm{~mm}$ for extension (C). Compared with the CM position in this dog when its pelvic limbs are in a neutral posture (B), the CM is cranially displaced when the dog's pelvic limbs are extended $(C)$ and caudally displaced when the pelvic limbs are flexed $(A)$. In all images, the dog's head is toward the left and dorsal is toward the top.

pelvic limbs in the neutral versus flexion, neutral versus extension, and flexion versus extension postures. The mean LSA per posture and the mean $\triangle$ LSA for postural changes from neutral to flexion, neutral to extension, and extension to flexion were determined for each dog. These values were then used for further analysis.

\section{CM translocation}

The caudalmost aspect of the CM was determined as the point where the signal intensity of the spinal cord appeared hypointense, compared with the hyperintense signal of the CSF. For dogs in which this point was not clearly distinguishable, transverse plane MRI images were analyzed for confirmation. To measure the location of the $\mathrm{CM}$, a line was first drawn from the center of the cranial endplate of $\mathrm{L} 7$ through the center of the caudal endplate of $\mathrm{L} 7$, then perpendicular to this craniocaudal line, a dorsoventral line was drawn to the caudal tip of the CM, and the distance between the level the cranial endplate of the L7 to the intersection of these 2 perpendicular lines was the measurement for the $\mathrm{CM}$ location (Figure 2). Displacement of the CM was defined as translocation of the CM cranially or caudally from its location in a dog imaged with its pelvic limbs positioned in neutral posture. A negative value indicated cranial displacement of the CM (ie, in extension posture), and a positive value indicated caudal displacement of the $\mathrm{CM}$ (ie, in flexion posture). Further, each dog's overall $\mathrm{CM}$ displacement was the measurement in the overall translocation of the CM identified for when the dog's pelvic limbs were in flexion versus extended posture. The mean CM location for each posture and the mean $\mathrm{CM}$ displacement for postural changes from neutral to flexion, neutral to extension, and extension to flexion were determined for each dog. These values were then used for further analysis.

To standardize CM displacement on the basis of dog body size, the mean CM displacement for each dog for each postural change (neutral to flexion posture, neutral to extension posture, and extension to flexion posture) was divided by the given dog's $\mathrm{L} 7$ vertebral body length to yield the dog's mean CM:L7. Additionally, the mean CM: $\triangle$ LSA, defined as the mean $\mathrm{CM}$ displacement per every $1^{\circ}$ of the mean $\triangle$ LSA was determined for each dog for each postural change. 


\section{Statistical analysis}

Statistical analyses were performed with available software. ${ }^{\mathrm{d}}$ A 2-way mixed model was used to evaluate intra- and interobserver reliability in agreement in measurements of the LSA and position of the $\mathrm{CM}$, and the ICCs were assessed. On the basis of the ICC value, agreement was considered poor (ICC, 0 to 0.5 ), moderate (ICC, 0.5 to 0.75 ), or excellent (ICC, 0.75 to 0.9$).{ }^{19}$ The Spearman rank correlation coefficient $(\rho)$ was used to assess for potential correlation between $\triangle$ LSA and CM displacement with correlation strength classified as very weak $(\rho, 0$ to 0.2$)$, weak ( $\rho$, 0.2 to 0.4 ), moderate ( $\rho, 0.4$ to 0.7$)$, strong ( $\rho, 0.7$ to 0.9 ), or very strong ( $\rho, 0.9$ to 1$).{ }^{19}$ Statistical analyses were conducted on the mean value for each variable for each dog. Values of $P<0.05$ were considered significant.

\section{Results}

\section{Animals}

There were 9 healthy adult Beagles in the study; 8 dogs were males ( 7 castrated and 1 sexually intact) and 1 was a sexually intact female. Mean \pm SD age was $31.3 \pm 10.1$ months (range, 21 to 42 months). Mean \pm SD body weight was $12.4 \pm 1.2 \mathrm{~kg}$ (range, 10.2 to $14.1 \mathrm{~kg}$ ). Each dog was considered healthy on the basis of findings from physical, neurologic, and radiographic examinations. None had clinical signs of lameness or back pain or radiographic evidence of an axial skeletal abnormality. The mean \pm SD duration of anesthesia for MRI was $2.11 \pm 0.4$ hours.

\section{LSA}

For measurements of the LSA, the interobserver ICC was excellent (0.991, 95\% CI, 0.986 to 0.996$)$, and the intra-observer ICCs for observer 1 and 2 were excellent $(0.999,95 \%$ CI, 0.998 to 1.000$)$. On the basis of results for dogs grouped by pelvic limb posture, the overall mean \pm SD LSA was $180.6 \pm 5.7^{\circ}$ for flexion posture, $156.8 \pm 5.6^{\circ}$ for neutral posture, and $148.3 \pm$ $4.2^{\circ}$ for extension posture (Table I). When changes in pelvic limb posture were considered, the overall mean \pm SD $\Delta$ LSA was $23.8 \pm 1.2^{\circ}$ for neutral to flexion posture, $-8.4 \pm 1.9^{\circ}$ for neutral to extension posture, and $32.2 \pm 2.2^{\circ}$ for extension to flexion posture.

\section{CM translocation}

For measurements of the CM location, the interobserver ICC was excellent $(0.968,95 \%$ CI, 0.934 to 0.986 ), and the intra-observer ICCs were excellent for observer 1 (0.986, 95\% CI, 0.970 to 0.994$)$ and for observer 2 ( $0.991,95 \% \mathrm{CI}, 0.978$ to 0.995$)$. When postural changes were considered, the overall mean \pm SD CM displacement was $9.09 \pm 3.29 \mathrm{~mm}$ for neutral to flexion posture, $-2.5 \pm 1.38 \mathrm{~mm}$ for neutral to extension posture, and $11.64 \pm 3.89 \mathrm{~mm}$ for extension to flexion posture (Table 2).

The $\Delta$ LSA strongly correlated $(\rho=0.705 ; 95 \% \mathrm{CI}$, 0.434 to $0.859 ; \mathrm{n}=9 ; P<0.05)$ with displacement of the CM. The overall mean CM: $\triangle$ LSA (ie, CM displacement for every $\left.1^{\circ} \Delta \mathrm{LSA}\right)$ grouped by postural changes was $0.39 \pm 0.14 \mathrm{~mm}$ for neutral to flexion posture, -0.32 $\pm 0.15 \mathrm{~mm}$ for neutral to extension posture, and $0.37 \pm$ $0.13 \mathrm{~mm}$ for extension to flexion posture (Table 2).

Table I-Mean \pm SD and range lumbosacral angle and CM location for 9 healthy Beagles that underwent dynamic MRI to obtain T2Wls of the lumbosacral region of the vertebral column with their pelvic limbs positioned in neutral, flexion, and extension postures.

\begin{tabular}{|c|c|c|c|c|c|c|}
\hline \multirow[b]{3}{*}{ Variable } & \multicolumn{6}{|c|}{ Pelvic limb posture } \\
\hline & \multicolumn{2}{|c|}{ Flexion } & \multicolumn{2}{|c|}{ Neutral } & \multicolumn{2}{|c|}{ Extension } \\
\hline & Mean \pm SD & Range & Mean \pm SD & Range & Mean \pm SD & Range \\
\hline $\begin{array}{l}\text { LSA }\left(^{\circ}\right) \\
\text { CM location }(\mathrm{mm})^{*}\end{array}$ & $\begin{aligned} 180.6 \pm 5.7 \\
* \quad 18.0 \pm 4.2\end{aligned}$ & $\begin{array}{c}168.6-188.5 \\
7.2-26.2\end{array}$ & $\begin{array}{r}156.8 \pm 5.6 \\
8.9 \pm 3.4\end{array}$ & $\begin{array}{c}145.8-166.7 \\
2.1-13.9\end{array}$ & $\begin{array}{r}148.3 \pm 4.2 \\
4.7 \pm 3.2\end{array}$ & $\begin{array}{c}140.1-153.5 \\
1.7-11.8\end{array}$ \\
\hline
\end{tabular}

*The level of the cranial endplate of the $L 7$ was used as the reference point for measurements.

Table 2-Mean \pm SD changes for variables of LSA and CM displacement on the basis of changes in pelvic limb posture from neutral to flexion or extension posture and from extension to flexion posture for the 9 dogs described in Table I.

\begin{tabular}{|c|c|c|c|c|c|c|}
\hline \multirow[b]{3}{*}{ Variable } & \multicolumn{6}{|c|}{ Change in pelvic limb posture } \\
\hline & \multicolumn{2}{|c|}{ Neutral to flexion } & \multicolumn{2}{|c|}{ Neutral to extension } & \multicolumn{2}{|c|}{ Extension to flexion } \\
\hline & Mean \pm SD & Range & Mean \pm SD & Range & Mean \pm SD & Range \\
\hline$\Delta \operatorname{LSA}\left({ }^{\circ}\right)$ & $23.8 \pm 1.2$ & 20.9 to 27.2 & $-8.4 \pm 1.9$ & -4.1 to -15.4 & $32.2 \pm 2.2$ & 28.4 to 36.4 \\
\hline CM displacement $(\mathrm{mm})^{*}$ & $9.09 \pm 3.29$ & 4.8 to 16.2 & $-2.5 \pm 1.38$ & -5.6 to -0.4 & $11.64 \pm 3.89$ & 5.50 to 18.80 \\
\hline CM: $\Delta$ LSA* $^{*}$ & $0.39 \pm 0.14$ & 0.20 to 0.60 & $-0.32 \pm 0.15$ & -0.47 to -0.53 & $0.37 \pm 0.13$ & 0.21 to 0.57 \\
\hline CM:L7* & $0.60 \pm 0.19$ & 0.31 to 0.85 & $-0.16 \pm 0.07$ & -0.27 to -0.04 & $0.76 \pm 0.23$ & 0.59 to 1.06 \\
\hline
\end{tabular}

*The level of the cranial endplate of the L7 was used as the reference point for measurements. Compared with the CM location for a dog in neutral posture, a negative value indicated cranial displacement of the CM (ie, in extension posture) and a positive value indicated caudal displacement of the CM (ie, in flexion posture). 
The overall mean CM:L7 (ie, CM displacement standardized to the length of the vertebral body of L7) was $0.60 \pm 0.19$ for neutral to flexion, $-0.16 \pm 0.07$ for neutral to extension, and $0.76 \pm 0.23$ for extension to flexion. Hence, a postural change from neutral to flexion posture of the pelvic limbs resulted in caudal displacement of the CM that was approximately 0.6 times the length of L7, and the overall CM displacement between extension and flexion postures was approximately 0.76 times the length of L7.

\section{Discussion}

In the present study, translocation of the CM in the vertebral column of dogs was evaluated in relation to pelvic limb posture: flexion, neutral, and extension. Our results indicated that as pelvic limb posture was changed from extension to flexion, the LSA increased and that the $\triangle \mathrm{LSA}$ positively correlated with CM displacement from its location in dogs positioned with their pelvic limbs in neutral posture. This finding supported our hypothesis that the $\mathrm{CM}$ displacement would correlate positively with the $\triangle$ LSA. However, caudal displacement of the CM varied greatly among dogs, with a mean change in CM location of 11.6 $\mathrm{mm}$ for dogs with their pelvic limbs extended versus flexed. When this finding was standardized to body size with the use of CM:L7, it corresponded to a change in the $\mathrm{CM}$ location equal to approximately 0.76 times the length of L7 in dogs of the present study.

Similarly, postural changes impact the location of the CM in people and have been used with the SLR test to diagnose muscle and spinal cord diseases in patients with lower back pain..$^{13-15,18,20}$ Also, similar to a study ${ }^{20}$ in human medicine, 1 study ${ }^{21}$ on dog cadavers confirmed strain to the sciatic nerve during the SLR test, and the authors reported that the pressure exerted on the sciatic nerve and the extent of hip joint flexion were relatively uniform and linearly correlated. Results of the present study provided quantitative data regarding translocation of the CM in the vertebral column and enhanced our understanding of the relationship between the soft tissue and bony structures of the lumbosacral region of the vertebral column and changes in this relationship on the basis of postural alterations. Further, it can be deduced that clinically normal translocation of the CM may not occur in the presence of restrictive disease, such as tethered cord syndrome.

Dogs have more flexible hip joints than do people; thus, the extent of hip joint flexion was greater in the dogs of the present study and was readily accommodated by the MRI system used. Because the neutral pelvic limb posture in dogs involves a minor flexion of the hip joints and stifle joint, we also chose to include complete hip joint extension as one of the postures. Our results indicated that the mean CM: $\triangle$ LSA was $0.37 \mathrm{~mm}$ of displacement of the CM per $1^{\circ}$ LLSA from extension to flexion. However, results varied substantially across dogs, and given the fact that the dogs were of the same breed and similar ages and weights, our results for CM: $\mathrm{LLSA}$ and $\mathrm{CM}: \mathrm{L} 7$ cannot be considered standard values in dogs.

A previous study ${ }^{8}$ shows that the position of the $\mathrm{CM}$ in dogs positioned with their pelvic limbs in a neutral posture was more cranial in heavier dogs. In that study, for dogs that weighed between 10 and $15 \mathrm{~kg}$, similar to the body weight of dogs in our study, the CM was located at a level that was in the cranial to the middle area of $\mathrm{L} 7$ when imaged in the neutral position, consistent with findings for the dogs of the present study. However, body weight of the dogs in our study was fairly uniform; thus, further research involving various breeds with more diverse body size and weight is needed to clarify whether substantial variations in body size and weight result in greater changes of the CM location with changes in posture.

In people with sciatic radiculopathy, pain originates primarily from the pressure exerted on the nerve roots resulting in reduced nerve gliding and severe tension on the nerve, thereby inducing decreased vascular flow, edema, and hypoxia. ${ }^{20}$ This mechanism also results in restricted movement of the spinal cord. ${ }^{13-15}$ The presence of nerve root pressure in lumbosacral disease may result in limited movement of the CM during postural changes. In people, there is reduced CM displacement in patients with IVD disease, radiculopathy, and lower back pain; yet, there is subsequent increased posterior CM movement in patients with improved clinical symptoms. ${ }^{16,17}$ The degree of pain and clinical signs in patients with lumbosacral disease could be explained by a similar mechanism; however, the degree of cauda equina compression does not often correlate with the severity of clinical signs (eg, pain and ataxia), and additional diagnostic procedures are needed to determine the underlying pathophysiologic processes to understand the prognosis in some patients with lumbosacral disease. ${ }^{22}$ Further work is needed in veterinary medicine to determine whether degenerative lumbosacral diseases (eg, vertebral stenosis or IVD extrusion) have similarly reduced CM translocation, and additional research could provide prognostic information and allow for the use of dynamic MRI to help in the differentiation between neurologic versus orthopedic signs of pain.

Given that there are limitations to the assessment of a dog's pain responses, the results of the present study and future work may also aid in evaluations after treatments. Dynamic lumbosacral MRI is currently used to diagnose tethered cord syndrome, and findings of the present study supported the usefulness of the procedure in being able to identify the lack of CM translocation during postural changes in tethered cord syndrome and similar, potentially underdiagnosed entities. ${ }^{10}$ Information about limited translocation of the CM in lumbosacral diseases other than with tethered cord syndrome is lacking in veterinary medicine, and the present study evaluated a relatively small part of the dynamic movements of the lumbosacral region; thus, additional research on this issue is warranted. 
Limitations of the present study included that our estimation of sagittal midline for selection of MRI images for evaluation could have been imprecise. Additionally, the number of dogs was small, and all dogs were of the same breed and similar size and body weight; therefore, the results may only be used as representative values for all dogs. Furthermore, the maximum nerve stretch induced by hip joint flexion and extension and the impact of stifle joint posture on CM translocation were not evaluated.

To our knowledge, MRI evidence of the presence of dynamic displacement of the CM during postural changes of the pelvic limbs of healthy dogs has not been reported previously. In the present study, we quantified the extent of the CM displacement with regard to $\triangle$ LSA for dogs undergoing MRI with their pelvic limbs positioned in extended, flexed, and neutral postures. Our findings suggested that when limited translocation of the $\mathrm{CM}$ is appreciated on dynamic MRI, veterinarians should suspect underlying lumbosacral pathophysiologic processes or anatomic abnormalities. Further, our results enhance the understanding of lumbosacral anatomic and physiologic characteristics in dogs and may help future comparative evaluations of the lumbosacral region of the vertebral column in dogs, the previous MRI foundation for which was limited to nondynamic MRI findings without the additional investigation of the effects of postural changes as we performed.

\section{Acknowledgments}

This manuscript represents a thesis submitted by Dr. Jihye Nam to the Seoul National University as partial fulfillment of the requirements for a Master of Science degree.

No external funding was used in this study. The authors declare that there were no conflicts of interest.

\section{Footnotes}

a. Hitachi AIRIS Vento, Hitachi Medical Co, Tokyo, Japan.

b. MR-QHC-101, Hitachi Medical Co, Tokyo, Japan.

c. INFINITT PACS, Infinitt Healthcare Co Ltd, Seoul, Korea.

d. SPSS Statistics, version 25.0 for Windows, IBM Corp, Armonk, NY.

\section{References}

1. Tobias KM, Johnston SA. Lumbosacral spine. In: Adin CA, Nelson RW, eds. Veterinary surgery small animal. Vol 1. St Louis: Elsevier Saunders, 2012;476-486.

2. Jones JC, Davies SE, Werre SR, et al. Effects of body position and clinical signs on L7-S1 intervertebral foraminal area and lumbosacral angle in dogs with lumbosacral disease as measured via computed tomography. Am J Vet Res 2008;69:1446-1454.

3. Wheeler SJ. Lumbosacral disease. Vet Clin North Am Small Anim Pract 1992;22:937-950.

4. Fletcher TF. Spinal cord and meninges. In: Evans HE, de Lahunta A, eds. Miller's anatomy of the dog. 4th ed. St Louis: Elsevier Saunders, 2013;589-610.
5. Parmer RH, Chambers JN. Canine lumbosacral disease. Part 1. Anatomy, pathophysiology, and clinical presentation. Compend Contin Educ Pract Vet 1991;13:61-69.

6. Saifuddin A, Burnett SJ, White J. The variation of position of the conus medullaris in an adult population. A magnetic resonance imaging study. Spine 1998;23:1452-1456.

7. Bergknut N, Meij B. Degenerative lumbosacral stenosis part 1 pathogenesis, clinical signs and diagnostics. Eur J Companion Anim Pract 2012;22:23-33.

8. Khan Z, Munro E, Shaw D, et al. Variation in the position of the conus medullaris and dural sac in adult dogs. Vet Rec 2019;185:20

9. Vermeulen HA. On the conus medullaris of the domestic animals, in Proceedings. KNAW 1915;780-792.

10. De Decker S, Watts V, Neilson DM. Dynamic lumbosacral magnetic resonance imaging in a dog with tethered cord syndrome with a tight filum terminale. Front Vet Sci 2017;4:134.

11. Sparks CR, Robertson I, Olby NJ. Morphometric analysis of spinal cord termination in Cavalier King Charles Spaniels. $J$ Vet Intern Med 2019;33:717-725.

12. Yamada S, Won DJ, Pezeshkpour G, et al. Pathophysiology of tethered cord syndrome and similar complex disorders. Neurosurg Focus 2007;23:E6.

13. Rade M, Könönen M, Vanninen R, et al. 2014 young investigator award winner: in vivo magnetic resonance imaging measurement of spinal cord displacement in the thoracolumbar region of asymptomatic subjects: part 1: straight leg raise test. Spine 2014;39:1288-1293.

14. Rade M, Könönen M, Vanninen R, et al. 2014 young investigator award winner: in vivo magnetic resonance imaging measurement of spinal cord displacement in the thoracolumbar region of asymptomatic subjects: part 2: comparison between unilateral and bilateral straight leg raise tests. Spine 2014;39:1294-1300.

15. Rade M, Shacklock M, Könönen M, et al. Part 3: developing methods of in vivo MRI measurement of spinal cord displacement in the thoracolumbar region of asymptomatic subjects with unilateral and bilateral straight leg raise tests. Spine 2015;40:935-941.

16. Rade M, Pesonen J, Könönen $\mathrm{M}$, et al. Reduced spinal cord movement with the straight leg raise test in patients with lumbar intervertebral disc herniation. Spine 2017;42:1117-1124.

17. Pesonen J, Rade M, Könönen M. Normalization of spinal cord displacement with the straight leg raise and resolution of sciatica in patients with lumbar intervertebral disc herniation: a 1.5-year follow-up study. Spine 2019;44:1064-1077.

18. Rebain R, Baxter GD, McDonough S. A systematic review of the passive straight leg raising test as a diagnostic aid for low back pain (1989 to 2000). Spine (Phila Pa 1976) 2002;27:E388-E395.

19. Rovai AP, Baker JD, Ponton MK. Social science research design and statistics: a practitioner's guide to research methods and IBM SPSS. 2nd ed. Chesapeake, Va: Watertree Press LLC, 2013;375.

20. Kobayashi S, Suzuki Y, Asai T, et al. Changes in nerve root motion and intraradicular blood flow during intraoperative femoral nerve stretch test. Report of four cases. J Neurosurg 2003;99:298-305

21. Babbage CS, Coppieters MW, McGowan CM. Strain and excursion of the sciatic nerve in the dog: biomechanical considerations in the development of a clinical test for increased neural mechanosensitivity. Vet J 2007;174:330-336.

22. Mayhew PD, Kapatkin AS, Wortman JA, et al. Association of cauda equina compression on magnetic resonance images and clinical signs in dogs with degenerative lumbosacral stenosis. J Am Anim Hosp Assoc 2002;38:555-562. 